•生物编目・2020年新物种专题

\title{
2020年中国双翅目新分类阶元
}

\author{
张冰，王亮，丁双玫，杨定〔*
}

中国农业大学植物保护学院, 北京 100193

摘要: 本文总结了2020年中国双翅目分类研究的进展以及分类学者们对双翅目分类的科学贡献, 并对中国双翅目新分类单 元的种类、分布地、发表期刊以及发表新分类单元数量的学者进行了统计。基于统计得到的中国双翅目标本2020年发表的3 新属113新种, 本研究发现果蝇科(Drosophilidae) (31新种)、缟蝇科(Lauxaniidae) (17新种)和摇蚊科(Chironomidae) (17新种)新 分类单元最多; 云南(43新种)、西藏(19新种或新属)和四川(17新种或新属)发现的新分类单元最多; 发表新分类单元最多的期 刊为ZooKeys (30新种或新属)、Zootaxa (27新种或新属) 和《昆虫分类学报》(22新种或新属); 发表新分类单元最多的双翅目 学者分别为华南农业大学陈宏伟团队、中国农业大学杨定团队和南开大学王新华团队。

关键词: 分类学; 新分类单元; 分类学者; 双翅目; 中国

张冰，王亮，丁双玫，杨定 (2021) 2020年中国双翅目新分类阶元. 生物多样性, 29, 1040-1043. doi: 10.17520/biods.2021183.

Zhang B, Wang L, Ding SM, Yang D (2021) New taxa of Diptera from China in 2020. Biodiversity Science, 29, 1040-1043. doi: 10.17520/biods.2021183.

\section{New taxa of Diptera from China in 2020}

\author{
Bing Zhang ${ }^{\mathbb{D}}$, Liang Wang, Shuangmei Ding, Ding Yang ${ }^{(\mathbb{D} *}$ \\ College of Plant Protection, China Agricultural University, Beijing 100193
}

\section{ABSTRACT}

Aims: In order to outline the contributions and advances of Diptera taxonomy in China in 2020, we summarize the detailed information of the new taxa, species distribution, and publication cases.

Methods: Potential source articles were searched and identified from online resources. The Chinese National Knowledge Infrastructure, Web of Science, Elsevier, Springer, and other related academic literature index databases were searched.

Results: Totally three new genera and 113 new species of Diptera were reported from China in 2020. We ranked the publications from different perspectives: the most newly described taxa were found in Drosophilidae (31 new species), Lauxaniidae (17 new species), and Chironomidae (17 new species); the regions with the greatest number of new taxa were Yunnan (43 new species), Xizang (19 new species or genera) and Sichuan (17 new species or genera); the journals with the most published new taxa are ZooKeys (30 new species or genera), Zootaxa (27 new species or genera) and Entomotaxonomia (22 new species or genera); The research groups that published the most new taxa are Hongwei Chen, Ding Yang, and Xinhua Wang.

Conclusion: Some progress has been made in the Chinese Diptera classification in 2020, however, gathering a deeper understanding of the dipteran taxonomy remains challenging due to the relatively short research history and insufficient and uneven research foundation. Diptera is a major order of insects, with about 150,000 described species and perhaps more than a quarter of a million species. According to our statistics, 113 new species were described in China last year, then it can be seen that the taxonomic research of Diptera in China has a long way to go. More professionals, more taxonomic group coverage, and wider international collaboration would contribute to the Chinese Diptera research to a higher level.

Key words: taxonomy; new taxon; taxonomist; Diptera; China

收稿日期: 2021-05-10; 接受日期: 2021-07-05

* 通讯作者 Author for correspondence. E-mail: dyangcau@126.com 
双翅目昆虫是世界上种类最为繁多的类群之 一, 作为昆虫纲四大目之一, 它们分布极其广泛, 除了深海等极端环境外几乎遍布世界七大洲的各 个角落。据统计, 已被描述的双翅目昆虫包含 200 多个科的159,294 个现生物种以及3,817个化石种, 分别隶属于 2 个主要的亚目一长角亚目 (Nematocera)和短角亚目 (Brachycera) (Pape et al, 2011)。其中短角亚目昆虫较为进化, 也是更为复杂 多样的类群之一。

双翅目昆虫食性各异, 有植食性、肉食性、腐 食性和寄生性等。虽然双翅目昆虫体型较小, 并不 起眼, 但它们对于人类的生产生活却有重要的影 响。其中传粉昆虫包括蚜蝇科(Syrphidae)和蜂虹科 (Bombyliidae)等, 由于在生态环境中的重要作用, 近年来尤其受到科学界的关注(Wu \& Zheng 2019); 以实蝇科(Tephritidae)和潜蝇科(Agromyzidae)为代 表的农业害虫每年都会给农业生产造成巨大损失; 卫生昆虫蚊科(Culicidae)和蝇科(Muscidae)的一些 种类甚至造成世界上每年超过 200 万人死亡 (Ssymank et al, 2008; Peng et al, 2020; Yang et al, 2020b)。本文总结了2020年中国双翅目分类学方面 的研究情况, 包括中国双翅目分类研究的进展以及 成果。

\section{数据来源}

本研究通过检索工具搜索关键字或关键词得 到目的资料, 再根据期刊(表2)具体文献进行数据统 计。关键字或关键词有: 2020、中国(China)、200多 个双翅目(亚)科的中文名和拉丁名、分类阶元 (taxa)、新属(new genus)和新种(new species)等。文 献检索工具包括中国知网 (https://www.cnki.net/)、

Web of Science (http://www.webofscience.com/)、 Elsevier (https://www.elsevier.com/)和Springer (https: //link.springer.com/)等。

\section{2 结果}

\subsection{0 年中国双翅目新分类单元}

如表1所示, 2020年发表了中国双翅目116个新 分类单元, 包括 3 个新属和 113 个新种, 隶属于 16 个 科。这16个科中, 有 3 个科包含 10 个以上的新分类单 元, 分别是果蝇科(Drosophilidae) (31)、缟蝇科 (Lauxaniidae) (17)和摇蚊科(Chironomidae) (17)。以
表1 2020年中国双翅目新分类单元

Table 1 List of new taxa of Diptera from China in 2020

\begin{tabular}{|c|c|c|c|}
\hline $\begin{array}{l}\text { 科(全世界数量*) } \\
\text { Families (Total number around } \\
\text { the world*) }\end{array}$ & $\begin{array}{l}\text { 新属 } \\
\text { New genus }\end{array}$ & $\begin{array}{l}\text { 新种 } \\
\text { New species }\end{array}$ & $\begin{array}{l}\text { 总数 } \\
\text { Total }\end{array}$ \\
\hline 果蝇科 Drosophilidae $(4,017)$ & & 31 & 31 \\
\hline 缟蝇科 Lauxaniidae $(1,900)$ & & 17 & 17 \\
\hline 摇蚊科 Chironomidae $(7,290)$ & & 17 & 17 \\
\hline 大蚊科 Tipulidae $(4,334)$ & & 7 & 7 \\
\hline 瘘蚊科 Cecidomyiidae $(6,296)$ & 1 & 6 & 7 \\
\hline 沼大蚊科 Limoniidae $(10,732)$ & & 6 & 6 \\
\hline 蝇科 Muscidae $(5,218)$ & & 5 & 5 \\
\hline 长足虹科 Dolichopodidae $(7,358)$ & & 4 & 4 \\
\hline 狂蝇科 Oestridae (176) & & 4 & 4 \\
\hline 舞虹科 Empididae $(3,142)$ & 1 & 3 & 4 \\
\hline 蚤蝇科 Phoridae $(4,202)$ & 1 & 3 & 4 \\
\hline 蠓科 Ceratopogonidae $(5,902)$ & & 3 & 3 \\
\hline 蚜蝇科 Syrphidae $(6,107)$ & & 3 & 3 \\
\hline 寄蝇科 Tachinidae $(9,626)$ & & 2 & 2 \\
\hline 尖尾蝇科 Lonchaeidae (504) & & 1 & 1 \\
\hline 麻蝇科 Sarcophagidae $(3,094)$ & & 1 & 1 \\
\hline 总计 Total & 3 & 113 & 116 \\
\hline
\end{tabular}

* Pape et al, 2011; Oosterbroek 2021

上 3 个科的新分类单元总数占 16 个科新分类单元总 数的 $56.03 \%$, 其余 13 个科新分类单元均在 10 个以下, 总数占 $43.97 \%$ 。

\subsection{0 年中国双翅目新分类单元发表期刊}

如表2所示, 116 个新分类单元分别发表在 14 个 国内外期刊上。有 4 个期刊发表了 10 个以上的新分 类单元, 分别是ZooKeys (30)、Zootaxa (27)、《昆虫 分类学报》(22)和Zoological Journal of the Linnean Society (17)。以 4 个期刊的新分类单元总数占新分 类单元总数的 $82.76 \%$, 其余 10 个期刊新分类单元均 在 10 个以下, 总数占 $17.24 \%$ 。

\subsection{0 年中国双翅目新分类单元各省区分布}

如表3所示, 116 个新分类单元分布在我国21个 省、市及自治区。有 4 个省区分布了 10 个以上的新 分类单元, 分别是云南(43)、西藏(19)、四川(17)和 浙江(11)。

\subsection{0 年中国双翅目新分类单元团队或学者贡献}

如表4所示, 2020年发表了中国双翅目116个新 分类单元, 分别由国内外专家团队或学者完成。有 3 
表2 2020年中国双翅目新分类单元发表期刊列表

Table 2 Publications list of new taxa of Diptera from China in 2020

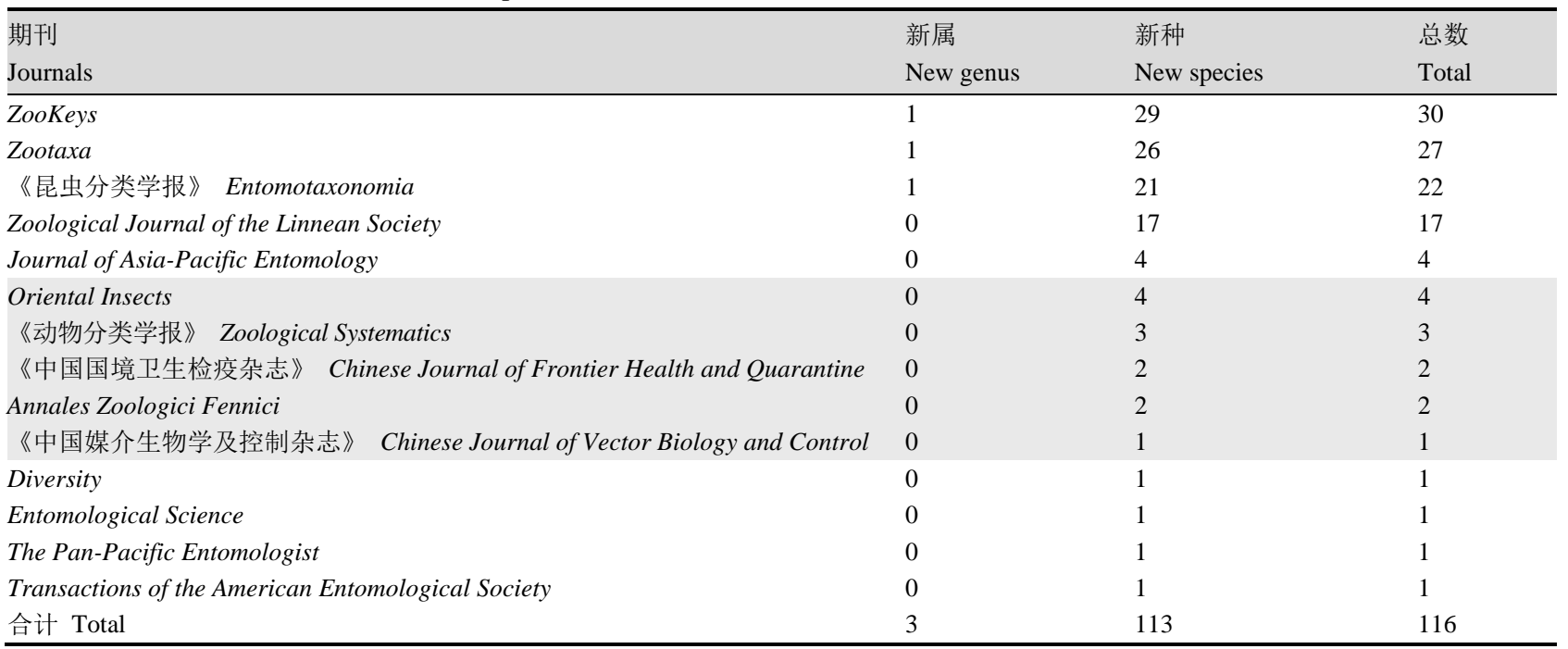

表3 2020年中国双翅目新分类单元各省/区列表

Table 3 Provinces(City or Autonomous region)list of new taxa of Diptera from China in 2020

\begin{tabular}{|c|c|c|c|}
\hline $\begin{array}{l}\text { 省(市或自治区) } \\
\text { Province/region }\end{array}$ & $\begin{array}{l}\text { 新属 } \\
\text { New genus }\end{array}$ & $\begin{array}{l}\text { 新种 } \\
\text { New species }\end{array}$ & $\begin{array}{l}\text { 总数 } \\
\text { Total }\end{array}$ \\
\hline 云南 Yunnan & & 43 & 43 \\
\hline 西藏 Xizang & 1 & 18 & 19 \\
\hline 四川 Sichuan & 1 & 16 & 17 \\
\hline 浙江 Zhejiang & & 11 & 11 \\
\hline 海南 Hainan & & 6 & 6 \\
\hline 台湾 Taiwan & & 4 & 4 \\
\hline 贵州 Guizhou & & 4 & 4 \\
\hline 广西 Guangxi & & 4 & 4 \\
\hline 陕西 Shaanxi & 1 & 3 & 4 \\
\hline 辽宁 Liaoning & 1 & 3 & 4 \\
\hline 宁夏 Ningxia & & 3 & 3 \\
\hline 河北 Hebei & 1 & 2 & 3 \\
\hline 黑龙江 Heilongjiang & & 2 & 2 \\
\hline 内蒙古 Inner Mongolia & & 2 & 2 \\
\hline 广东 Guangdong & & 2 & 2 \\
\hline 青海 Qinghai & & 2 & 2 \\
\hline 北京 Beijing & 1 & 1 & 2 \\
\hline 福建 Fujian & & 1 & 1 \\
\hline 甘肃 Gansu & & 1 & 1 \\
\hline 江西 Jiangxi & & 1 & 1 \\
\hline 河南 Henan & & 1 & 1 \\
\hline 合计 Total & $3^{*}$ & 113 & 116 \\
\hline
\end{tabular}

* 新属Pekinomyia分布在多个省份 The new genus is distributed in multiple provinces

个专家团队发表了 10 个以上的新分类单元，分别是 华南农业大学陈宏伟团队、中国农业大学杨定团队 和南开大学王新华团队。以上 3 个团队的新分类单
表4 2020年中国双翅目命名10个以上新分类单元的学者 Table 4 List of scholars that named the new taxa for over 10 of Diptera from China in 2020

\begin{tabular}{|c|c|c|c|c|c|}
\hline & $\begin{array}{l}\text { 陈宏伟 } \\
\text { Hongwei } \\
\text { Chen }\end{array}$ & $\begin{array}{l}\text { 杨定 } \\
\text { Ding } \\
\text { Yang }\end{array}$ & $\begin{array}{l}\text { 王亚莲 } \\
\text { Yalian } \\
\text { Wang }\end{array}$ & $\begin{array}{l}\text { 李文亮 } \\
\text { Wenliang } \\
\text { Li }\end{array}$ & $\begin{array}{l}\text { 王新华 } \\
\text { Xinhua } \\
\text { Wang }\end{array}$ \\
\hline $\begin{array}{l}\text { 果蝇科 } \\
\text { Drosophilidae }\end{array}$ & 29 & & 24 & & \\
\hline $\begin{array}{l}\text { 缟蝇科 } \\
\text { Lauxaniidae }\end{array}$ & & 16 & & 16 & \\
\hline $\begin{array}{l}\text { 摇蚊科 } \\
\text { Chironomidae }\end{array}$ & & & & & 11 \\
\hline $\begin{array}{l}\text { 大蚊科 } \\
\text { Tipulidae }\end{array}$ & & 2 & & & \\
\hline $\begin{array}{l}\text { 沼大蚊科 } \\
\text { Limoniidae }\end{array}$ & & 2 & & & \\
\hline $\begin{array}{l}\text { 长足虹科 } \\
\text { Dolichopodidae }\end{array}$ & & 4 & & & \\
\hline $\begin{array}{l}\text { 舞虬科 } \\
\text { Empididae }\end{array}$ & & $4^{*}$ & & & \\
\hline 合计 Total & 29 & $28^{*}$ & 24 & 16 & 11 \\
\hline
\end{tabular}

* 新属及新种 New genus and new species

元总数占整个双翅目分类学者发表的新分类单元 总数的 $58.62 \%$, 其余双翅目分类团队或学者发表的 新分类单元均在 10 个以下，总数占 $41.37 \%$ 。

\section{3 小结}

中国双翅目分类工作主要集中在数量较大的 类群上，例如果蝇科和摇蚊科等，而大部分数量较 小类群的分类工作相对贵乏，例如狂蝇科和尖尾蝇 科等，且大部分数量小的类群多年并无新的报道和 研究, 加之中国双翅目分类学者也相对较少, 因此 
大量的双翅目新分类单元有待发现 (Yang et al, 2020a，b)。从发表新分类单元的期刊来看, 分类工 作者们主要把成果发表在传统分类学期刊上, 发表 在其他期刊上的新分类单元相对较少, 这也说明了 分类学工作与其他领域的结合相对不足。随着科学 技术的进步与发展, 传统分类工作也需要更多的跨 学科和跨领域的交流合作, 这对传统分类工作是一 种挑战, 但也是新的突破和机遇。从新分类单元的 分布情况不难看出, 我国西南地区的新分类单元占 绝大多数, 这和我国整体的气候、植被类型以及其 他多种自然和人文因素密不可分。西南地区多样的 自然环境条件孕育了丰富的物种资源, 成为生物多 样性分化和研究热点地区。

尽管2020年中国双翅目分类工作取得了一定 的进展, 但基础差、研究历史短、本底不清等仍是 阻碍该领域快速发展的绊脚石。作为昆虫纲四大目 之一的双翅目如果以每年发表100多种的速度来计 算, 要基本调查清楚中国双翅目昆虫的本底情况还 需更多时日, 任重而道远!

致谢: 非常感谢华南农业大学陈宏伟教授团队、南 开大学王新华教授团队以及其他双翅目分类团队 和学者们为本研究提供的原始数据。特别感谢为本 研究提供图片的昭通市绿色食品发展中心石安宪 先生。

\section{ORCID}

张冰 (D) https://orcid.org/0000-0002-3996-1760

杨定 (i) https://orcid.org/0000-0002-7685-3478

\section{参考文献}

Oosterbroek P (2021) Catalogue of the Craneflies of the World (Diptera, Tipuloidea, Pediciidae, Limoniidae, Cylindrotomidae, Tipulidae). https://ccw.naturalis.nl/index.php. (accessed on 2021-06-22)

Pape T, Blagoderov V, Mostovski MB (2011) Order Diptera Linnaeus, 1758. Zootaxa, 3148, 222-229.

Peng W, Feng MJ, Chen H, Han BY (2020) Progress on genome sequencing of Dipteran insects. Hereditas (Beijing), 42, 1093-1109. (in Chinese with English abstract) [彭威, 冯蒙洁, 陈皓, 韩宝瑜 (2020) 双翅目昆虫基因组研究进 展. 遗传, 42, 1093-1109.]

Ssymank A, Kearns CA, Pape T, Thompson FC (2008) Pollinating Flies (Diptera): A major contribution to plant diversity and agricultural production. Biodiversity, 9, 86-89.

Wu PF, Zheng G (2019) Progress in pollination by Dipteran insects. Acta Entomologica Sinica, 62, 516-526. (in Chinese with English abstract) [武鹏峰, 郑国 (2019) 双翅目昆虫 传粉研究进展. 昆虫学报, 62, 516-526.]

Yang D, Li Z, Liu QF (2020a) Species Catalog of China. Vol. 2. Animals, Insecta (V), Diptera (1), Nematocera. Science Press, Beijing. (in Chinese) [杨定, 李竹, 刘启飞 (2020a) 中国生物物种名录第二卷: 动物, 昆虫 $(\mathrm{V})$ 双翅目(1)长角 亚目. 科学出版社, 北京.]

Yang D, Wang MQ, Li WL (2020b) Species Catalog of China. Vol. 2. Animals, Insecta (VII), Diptera (3), Brachycera Cyclorrhaphous. Science Press, Beijing. (in Chinese) [杨定, 王孟卿, 李文亮 (2020b) 中国生物物种名录(第二卷): 动 物, 昆虫(VII)双翅目(3)短角亚目, 蝇类. 科学出版社, 北 京.]

\section{附录 Supplementary Material}

附录1 2020年中国双翅目新分类单元文献索引

Appendix 1 Bibliography of new taxa of Diptera from China in 2020

http://www.biodiversity-science.net/fileup/PDF/2021183-1.pdf

(责任编委: 白明 责任编辑: 时意专) 


\section{0年中国双翅目新分类单元文献目录}

Bibliography of New Taxa of Chinese Diptera, 2020 


\section{目录 Contents}

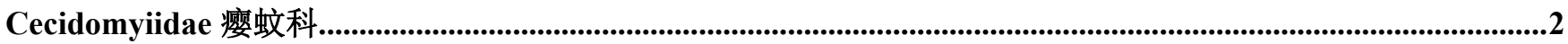

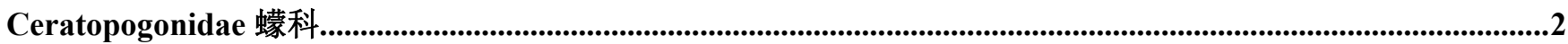

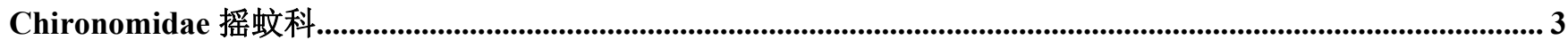

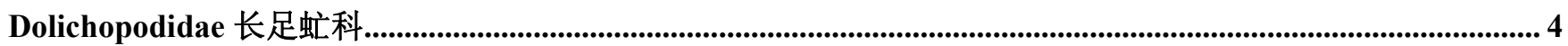

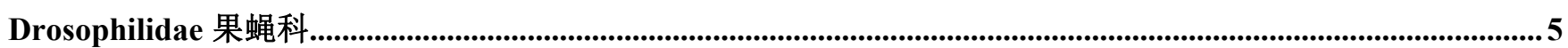

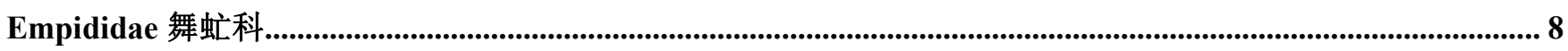

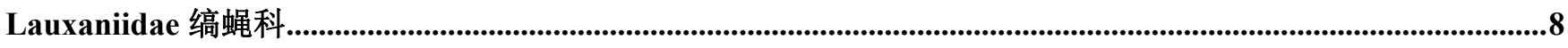

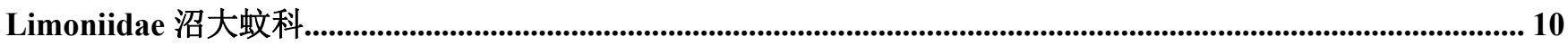

Lonchaeidae 尖尾蝇科............................................................................................................................................................................... 10

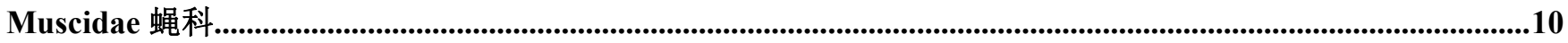

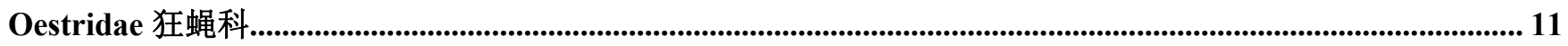

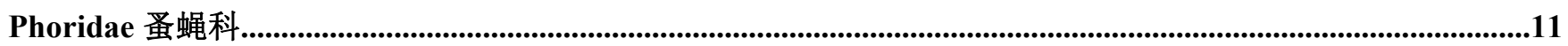

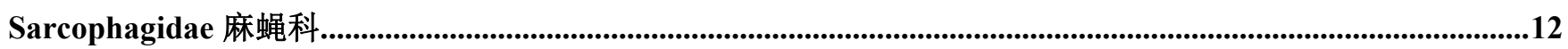

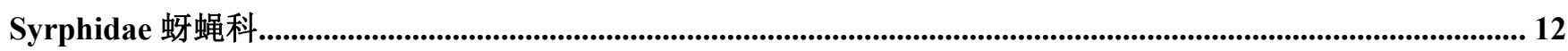

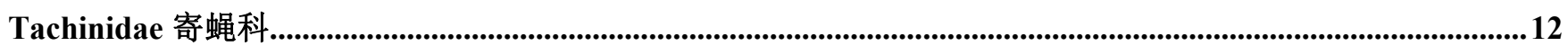

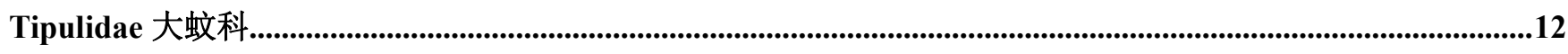

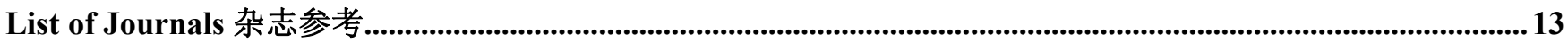




\section{Cecidomyiidae 瘦蚊科}

Bruggmanniella litseae S. F. Lin, M. M. Yang \& M. Tokuda [New Species] IUCN: NE; Evidence: Morphology; COI

LIN Sheng-feng, TOKUDA Makoto, YANG Man-miao, Leaf gall polymorphism and molecular phylogeny of a new Bruggmanniella species (Diptera: Cecidomyiidae: Asphondyliini) associated with Litsea acuminata (Lauraceae) in Taiwan, with ecological comparisons and a species description. Entomological Science 23: 10-22. 2020 [13] doi: 10.1111/ens.12391

CHINA (Taiwan*)

Gephyraulus lycantha K. L. Jiao \& P. Kolesik [New Species] IUCN: NE; Evidence: Morphology

JIAO Ke-long, ZHOU Xiao-yi, QIAO Hai-li, WANG Hao, CHEN Jun, LIU Bai-ming, BU Wen-jun, KOLESIK Peter, A new species of gall midge (Diptera: Cecidomyiidae) damaging flower buds of goji berry Lycium barbarum (Solanaceae). Journal of Asia-Pacific Entomology 23 (4): 930-934. 2020 [931] doi: doi.org/10.1016/j.aspen.2020.07.020

CHINA (Ningxia*)

Rhizomyia acroleptosipha K. L. Jiao, X. Y. Zhou \& W. J. Bu 端细管根瘦蚊 [New Species] IUCN: NE; Evidence: Morphology

DU Jing, ZHOU Xiao-yi, JIAO Ke-long, WANG Yuanhong, BU Wen-jun, A new species of Rhizomyia Kieffer (Diptera: Cecidomyiidae) from Northeast China. Entomotaxonomia 42 (3): 194-199. 2020 [196] doi: 10.11680/entomotax.2020018

CHINA (Heilongjiang*)

Pararhizomyia dolichostylata K. L. Jiao, H. Wang \& W. J. Bu [New Species] IUCN: NE; Evidence: Morphology

JIAO Ke-long, WANG Hao, BU Wen-jun, Pararhizomyia Mamaev (Diptera: Cecidomyiidae) from China. Oriental $\begin{array}{lllll}\text { Insects } & 54: 1 \quad 75-87 . & 2020 \quad \text { [79] doi: }\end{array}$ 10.1080/00305316.2019.1580626

CHINA (Yunnan*)

Pekinomyia K. L. Jiao \& P. Kolesik [New Genus] IUCN: NE; Evidence: Morphology; COI, 12S

JIAO Ke-long, ZHOU Xiao-yi, WANG Hao, FU Huai-jun, ZHOU Da-kang, LI Jing-bo, XIONG De-ping, WANG Yuan-hong, BU Wen-jun, KOLESIK Peter, A new genus and species of gall midge (Diptera: Cecidomyiidae) inducing leaf galls on Peking lilac, Syringa reticulata subsp. pekinensis (Oleaceae), in China. Zootaxa 4742 (1): 194-200. 2020 [195] doi: $10.11646 /$ zootaxa.4742.1.13

CHINA (Beijing*)

Pekinomyia syringae K. L. Jiao \& P. Kolesik [New Species] IUCN: NE; Evidence: Morphology; COI, 12S

JIAO Ke-long, ZHOU Xiao-yi, WANG Hao, FU Huai-jun, ZHOU Da-kang, LI Jing-bo, XIONG De-ping, WANG Yuan-hong, BU Wen-jun, KOLESIK Peter, A new genus and species of gall midge (Diptera: Cecidomyiidae) inducing leaf galls on Peking lilac, Syringa reticulata subsp. pekinensis (Oleaceae), in China. Zootaxa 4742 (1): 194-200. 2020 [195] doi: $10.11646 /$ zootaxa.4742.1.13

CHINA (Beijing*)

Schizomyia maesae A. K. Elsayed, S. F. Lin \& T. Tokuda [New Species] IUCN: NE; Evidence: Morphology

ELSAYED Ayman-khamis, LIN Sheng-feng, YANG Manmiao, TOKUDA Makoto, The first report of the genus Schizomyia (Diptera: Cecidomyiidae) in Taiwan, with description of a new species forming stem galls on Maesa perlaria var. formosana (Primulaceae). Journal of AsiaPacific Entomology 23 (4): 1083-1088. 2020 [1084] doi: 10.1016/j.aspen.2020.08.011

CHINA (Taiwan*)

\section{Ceratopogonidae 蠓科}

Culicoides (Beltranmyia) beishanensis Y. J. Li \& G. P. Liu 北山 库蠓 [New Species] IUCN: NE; Evidence: Morphology

LI Yong-jiu, LYU Cheng-liang, XING Tian-yao, LIU Guoping, Fauna of hematophagous midges and a new species of Culicoides (Beltranmyia) in Raohe port of Heilongjiang province. Chinese Journal of Frontier Health and Quarantine 43 (3): 174-176. 2020 [175] doi: 10.16408/j.1004 $-9770.2020 .03 .007$

CHINA (Heilongjiang*)

Culicoides (Oecacta) hekouensis X. Zhou, J. Yang \& G. P. Liu 河口库蠓 [New Species] IUCN: NE; Evidence: Morphology

ZHOU Xu, YANG Jun, LIU Guo-ping, Description of a new species Culicoides (Oecacta) Diptera: Ceratopogonidae in China. Chinese Journal of Frontier Health and Quarantine 43 (6): 408-409. 2020 [408] doi: 10.16408/j.1004- 
9770.2020.06.009

CHINA (Yunnan*)

Dasyhelea jinshajangensis J. P. Chen, W. P. Qian \& Y. X. Yu 金沙江毛蠓 [New Species] IUCN: NE; Evidence: Morphology

HAN Shi-kang, BIAN Shao-yong, WANG Song-lin, CHEN Jian-ping, LIU Yu, WANG Pei-zhang, DENG Yong, QIAN Wei-ping, YU Yi-xin, A new species and a new record of midges in Sichuan province, China (Diptera: Ceratopogonidae). Chinese Journal of Vector Biology and Control 31 (4): 462-464. 2020 [463] doi: $10.11853 /$ j.issn. 1003.8280 .2020 .04 .017

CHINA (Sichuan*)

\section{Chironomidae 摇蚊科}

Amnihayesomyia subflava H. Q. Tang \& H. Niitsuma [New Species] IUCN: NE; Evidence: Morphology

TANG Hong-qu, NIITSUMA Hiromi, Review of the genus Amnihayesomyia Niitsuma (Diptera: Chironomidae: Tanypodinae), with descriptions of three new species from China. Zootaxa 4743 (3): 411-418. 2020 [416] doi: 10.11646/zootaxa.4743.3.8

CHINA (Xizang*)

Amnihayesomyia tibetica H. Q. Tang \& H. Niitsuma [New Species] IUCN: NE; Evidence: Morphology

TANG Hong-qu, NIITSUMA Hiromi, Review of the genus Amnihayesomyia Niitsuma (Diptera: Chironomidae: Tanypodinae), with descriptions of three new species from China. Zootaxa 4743 (3): 411-418. 2020 [415] doi: 10.11646/zootaxa.4743.3.8

CHINA (Xizang*)

Amnihayesomyia vittata $\mathrm{H}$. Q. Tang \& H. Niitsuma [New Species] IUCN: NE; Evidence: Morphology

TANG Hong-qu, NIITSUMA Hiromi, Review of the genus Amnihayesomyia Niitsuma (Diptera: Chironomidae: Tanypodinae), with descriptions of three new species from China. Zootaxa 4743 (3): 411-418. 2020 [413] doi: 10.11646/zootaxa.4743.3.8

CHINA (Xizang*,Yunnan )

Corynoneura aggeris $\mathrm{Y} . \mathrm{Fu}$ [New Species] IUCN: NE; Evidence: Morphology
FU Yue, WANG Xin-hua, FANG Xiang-Liang, XIAO Yun-li, FU Jun, LIN Xiao-long, Corynoneura Winnertz (Diptera, Chironomidae, Orthocladiinae) from Zhejiang Province, China. Zootaxa 4890 (1): 83-96. 2020 [84] doi: 10.11646/zootaxa.4890.1.4

CHINA (Zhejiang*)

Corynoneura lateralis $\mathrm{Y} . \mathrm{Fu}$ [New Species] IUCN: NE; Evidence: Morphology

FU Yue, WANG Xin-hua, FANG Xiang-Liang, XIAO Yun-li, FU Jun, LIN Xiao-long, Corynoneura Winnertz (Diptera, Chironomidae, Orthocladiinae) from Zhejiang Province, China. Zootaxa 4890 (1): 83-96. 2020 [86] doi: 10.11646/zootaxa.4890.1.4

CHINA (Zhejiang*)

Corynoneura petitspectabilis Y. Fu [New Species] IUCN: NE; Evidence: Morphology

FU Yue, WANG Xin-hua, FANG Xiang-Liang, XIAO Yun-li, FU Jun, LIN Xiao-long, Corynoneura Winnertz (Diptera, Chironomidae, Orthocladiinae) from Zhejiang Province, China. Zootaxa 4890 (1): 83-96. 2020 [89] doi: 10.11646/zootaxa.4890.1.4

CHINA (Zhejiang*)

Corynoneura prolata $\mathrm{Y} . \mathrm{Fu}$ [New Species] IUCN: NE; Evidence: Morphology

FU Yue, WANG Xin-hua, FANG Xiang-Liang, XIAO Yun-li, FU Jun, LIN Xiao-long, Corynoneura Winnertz (Diptera, Chironomidae, Orthocladiinae) from Zhejiang Province, China. Zootaxa 4890 (1): 83-96. 2020 [90] doi: 10.11646/zootaxa.4890.1.4

CHINA (Zhejiang*)

Corynoneura recta Y. Fu [New Species] IUCN: NE; Evidence: Morphology

FU Yue, WANG Xin-hua, FANG Xiang-Liang, XIAO Yun-li, FU Jun, LIN Xiao-long, Corynoneura Winnertz (Diptera, Chironomidae, Orthocladiinae) from Zhejiang Province, China. Zootaxa 4890 (1): 83-96. 2020 [92] doi: 10.11646/zootaxa.4890.1.4

CHINA (Zhejiang*)

Cryptotendipes bullum C. Song \& X. H. Wang [New Species] IUCN: NE; Evidence: Morphology

SONG Chao, ANDERSEN Trond, YAN Chun-cai, QI Xin, WANG Xin-hua, A new species of Cryptotendipes Beck \& Beck, 1969 (Diptera: Chironomidae) from Hainan Island, 
Oriental China. The Pan-Pacific Entomologist 96 (4): 253257. 2020 [254] doi: 10.3956/2020-96.4.253

CHINA (Hainan*)

Kiefferulus trigonum C. Song, X. H. Wang, W. J. Bu \& X. Qi [New Species] IUCN: NE; Evidence: Morphology; 18S, 28S, COI3P, CAD1, CAD4, PGD

SONG Chao, WANG Xin-hua, BU Wen-jun, QI Xin, Morphology lies: a case-in-point with a new non-biting midge species from Oriental China (Diptera, Chironomidae). ZooKeys 909: 67-77. 2020 [72] doi: 10.3897/zookeys.909.39347

CHINA (Fujian*, Guizhou, Guangxi, Hainan)

Limnophyes nudus C. Song, J. C. Zheng, X. H. Wang \& X. Qi [New Species] IUCN: NE; Evidence: Morphology; COI

SONG Chao, ZHENG Jia-cheng, WANG Xin-hua \& QI Xin, A new species of Limnophyes Eaton (Diptera: Chironomidae) from Xizang, China. Zootaxa 4722 (3): 295 300. 2020 [296] doi: 10.11646/zootaxa.4722.3.7

CHINA (Xizang*)

Macropelopia (Macropelopia) pergrandis H. Q. Tang \& H. Niitsuma [New Species] IUCN: NE; Evidence: Morphology

TANG Hong-qu, NIITSUMA Hiromi, Revision of the Chinese Macropelopia (Diptera: Chironomidae: Tanypodinae), with description of a new species. Zootaxa 4834 (2): 207-218. 2020 [208] doi:

10.11646/zootaxa.4834.2.3

CHINA (Yunnan*, Xizang, Sichuan)

Parachaetocladius squamula W. B. Liu \& W. Cao [New Species] IUCN: NE; Evidence: Morphology

LIU Wen-bin, CAO Wei, ZHAO Cong, YAN Chun-cai, Parachaetocladius squamula (Diptera, Chironomidae, Orthocladiinae), a new species from China. Annales Zoologici Fennici 57 (1): 145-149. 2020 [146] doi: doi.org/10.5735/086.057.0116

CHINA ( Zhejiang*)

Polypedilum (Cerobregma) paracyclus X. Qi \& C. Song [New Species] IUCN: NE; Evidence: Morphology; COI

QI Xin, SONG Chao, GE Kai-xuan, WANG Xin-hua, Polypedilum (Cerobregma) paracyclus sp. n., a new species from Oriental China (Diptera: Chironomidae). Zootaxa 4881 (1): 189-195. 2020 [191] doi: 10.11646/zootaxa.4881.1.12
CHINA (Guangxi*)

Rheocricotopus (Psilocricotopus) kongi X. L. Lin \& X. H. Wang [New Species] IUCN: NE; Evidence: Morphology; COI

LIN Xiao-long, YU Hai-jun, WANG Qian, BU Wen-jun \& WANG Xin-hua, DNA barcodes and morphology confirm a new species of Rheocricotopus (Psilocricotopus) orientalis group (Diptera: Chironomidae). Zootaxa 4768 (2): 282-290. 2020 [286] doi: 10.11646/zootaxa.4768.2.9

CHINA (Hainan*)

Thienemanniella dapanensis $\mathrm{Y}$. Fu [New Species] IUCN: NE; Evidence: Morphology

FU Yue, WANG Xin-hua, FANG Xiang-Liang, XIAO Yun-li, LIN Xiao-long, Thienemanniella dapanensis (Diptera: Chironomidae: Orthocladiinae), a new species from Zhejiang Province, China, and redescription of two Thienemanniella species. Annales Zoologici Fennici 57: 209-214. 2020 [210] doi: $10.5735 / 086.057 .0121$

CHINA (Zhejiang*)

Yaeprimus balteatus W. Han \& H. Q. Tang [New Species] IUCN: NE; Evidence: Morphology

HAN Wu, WEI Jin-xing, LIN Xiao-long, TANG Hong-qu, The Afro-Oriental Genus Yaeprimus Sasa et Suzuki (Diptera: Chironomidae: Chironomini): Phylogeny, New Species and Expanded Diagnoses. Diversity 12 (1): 31. 2020 [7] doi: 10.3390/d12010031

CHINA (Guangdong*, Hainan)

\section{Dolichopodidae 长足虬科}

Chrysotus shuensis R. S. Liu \& D. Yang 蜀小异长足 虻 [New Species] IUCN: NE; Evidence: Morphology

LIU Ruo-si, ZHONG Yong, ZHANG Rui-feng, YANG Ding, A study of Chrysotus (Diptera: Dolichopodidae) from Sichuan, China with one new species. Entomotaxonomia 42 (2): 116-121. 2020 [117] doi: 10.11680/entomotax.2020016 CHINA (Sichuan*)

Hercostomus liangi Qilemoge, L. L. Zhang \& D. Yang 亮氏寡 长足虻 [New Species] IUCN: NE; Evidence: Morphology

QILEMOGE, ZHANG Li-li, YANG Ding, A Key to Species Groups of the Genus Hercostomus (Diptera: Dolichopodidae) 
from China, with Description of One New Species. Transactions of the American Entomological Society 146: 305-321. 2020 [341] doi: 10.3157/061.146.0202

CHINA (Gansu*)

Rhaphium huzhuense Qilemoge, C. Lin \& D. Yang 互助雉长 足虻 [New Species] IUCN: NE; Evidence: Morphology

QILEMOGE, LIN Chen, QI Pei-feng, LI De-ming, YANG Ding, Two new species of Rhaphium from Qinghai Province, China (Diptera, Dolichopodidae, Rhaphiinae). ZooKeys 931: 73-84. 2020 [77] doi: 10.3897/zookeys.931.49671

CHINA (Qinghai*)

Rhaphium minhense Qilemoge, C. Lin \& D. Yang 民和锥长 足虻 [New Species] IUCN: NE; Evidence: Morphology

QILEMOGE, LIN Chen, QI Pei-feng, LI De-ming, YANG Ding, Two new species of Rhaphium from Qinghai Province, China (Diptera, Dolichopodidae, Rhaphiinae). ZooKeys 931: 73-84. 2020 [80] doi: 10.3897/zookeys.931.49671

CHINA (Qinghai*)

\section{Drosophilidae 果蝇科}

Amiota beama Y. L. Wang, H. L. Cao \& H. W. Chen [New Species] IUCN: NE; Evidence: Morphology

WANG Ya-lian, CAO Hui-luo, CHEN Hong-wei, Molecular phylogeny and species delimitation of Amiota alboguttata and Amiota basdeni species groups (Diptera: Drosophilidae) from East Asia. Zoological Journal of the Linnean Society 189 (4): 1370-1397. 2020 [1378] doi: 10.1093/zoolinnean/zlz132

CHINA (Yunnan*)

Amiota bifoliacea Y. L. Wang \& H. W. Chen 双叶阿果蝇 [New Species] IUCN: NE; Evidence: Morphology; COI

WANG Ya-lian, CHEN Hong-wei, Eleven new species in the genus Amiota (Diptera: Drosophilidae) from Southwest China. Entomotaxonomia 42 (3): 206-226. 2020 [220] doi: 10.11680/entomotax.2020024

CHINA (Yunnan*)
Amiota brevifoliacea Y. L. Wang \& H. W. Chen 短叶阿果蝇 [New Species] IUCN: NE; Evidence: Morphology; COI

WANG Ya-lian, CHEN Hong-wei, Eleven new species in the genus Amiota (Diptera: Drosophilidae) from Southwest China. Entomotaxonomia 42 (3): 206-226. 2020 [217] doi: 10.11680/entomotax.2020024

CHINA (Yunnan*)

Amiota cyclophylla Y. L. Wang, H. L. Cao \& H. W. Chen [New Species] IUCN: NE; Evidence: Morphology

WANG Ya-lian, CAO Hui-luo, CHEN Hong-wei, Molecular phylogeny and species delimitation of Amiota alboguttata and Amiota basdeni species groups (Diptera: Drosophilidae) from East Asia. Zoological Journal of the Linnean Society 189 (4): 1370-1397. 2020 [1380] doi: 10.1093/zoolinnean/zlz132

CHINA (Yunnan*)

Amiota flormontana Y. L. Wang, H. L. Cao \& H. W. Chen [New Species] IUCN: NE; Evidence: Morphology

WANG Ya-lian, CAO Hui-luo, CHEN Hong-wei, Molecular phylogeny and species delimitation of Amiota alboguttata and Amiota basdeni species groups (Diptera: Drosophilidae) from East Asia. Zoological Journal of the Linnean Society 189 (4): 1370-1397. 2020 [1381] doi: 10.1093/zoolinnean/zlz132 CHINA (Yunnan*)

Amiota geisson Y. L. Wang \& H. W. Chen 边寨阿果蝇 $[\mathrm{New}$ Species] IUCN: NE; Evidence: Morphology; COI

WANG Ya-lian, CHEN Hong-wei, Eleven new species in the genus Amiota (Diptera: Drosophilidae) from Southwest China. Entomotaxonomia 42 (3): 206-226. 2020 [221] doi: 10.11680/entomotax.2020024

CHINA (Yunnan*)

Amiota gigantomelania Y. L. Wang \& H. W. Chen 大黑阿果蝇

[New Species] IUCN: NE; Evidence: Morphology; COI

WANG Ya-lian, CHEN Hong-wei, Eleven new species in the genus Amiota (Diptera: Drosophilidae) from Southwest China. Entomotaxonomia 42 (3): 206-226. 2020 [211] doi: 10.11680/entomotax.2020024

CHINA (Yunnan*, Sichuan)

Amiota jianjuni Y. L. Wang, H. L. Cao \& H. W. Chen [New Species] IUCN: NE; Evidence: Morphology

WANG Ya-lian, CAO Hui-luo, CHEN Hong-wei, Molecular phylogeny and species delimitation of Amiota alboguttata and 
Amiota basdeni species groups (Diptera: Drosophilidae) from East Asia. Zoological Journal of the Linnean Society 189 (4): 1370-1397. 2020 [1386] doi: 10.1093/zoolinnean/zlz132

CHINA (Sichuan*)

Amiota medidehiscentia Y. L. Wang \& H. W. Chen 中裂阿果 蝇 [New Species] IUCN: NE; Evidence: Morphology; COI

WANG Ya-lian, CHEN Hong-wei, Eleven new species in the genus Amiota (Diptera: Drosophilidae) from Southwest China. Entomotaxonomia 42 (3): 206-226. 2020 [213] doi: 10.11680/entomotax.2020024

CHINA (Yunnan*)

Amiota multiprocessa Y. L. Wang \& H. W. Chen 多突阿果蝇 [New Species] IUCN: NE; Evidence: Morphology; COI

WANG Ya-lian, CHEN Hong-wei, Eleven new species in the genus Amiota (Diptera: Drosophilidae) from Southwest China. Entomotaxonomia 42 (3): 206-226. 2020 [214] doi: 10.11680/entomotax.2020024

CHINA (Yunnan*)

Amiota nigripes Y. L. Wang \& H. W. Chen 黑足阿果蝇 [New Species] IUCN: NE; Evidence: Morphology; COI

WANG Ya-lian, CHEN Hong-wei, Eleven new species in the genus Amiota (Diptera: Drosophilidae) from Southwest China. Entomotaxonomia 42 (3): 206-226. 2020 [215] doi: 10.11680/entomotax.2020024

CHINA (Yunnan*)

Amiota nulliseta Y. L. Wang \& H. W. Chen 光叶阿果蝇 [New Species] IUCN: NE; Evidence: Morphology; COI

WANG Ya-lian, CHEN Hong-wei, Eleven new species in the genus Amiota (Diptera: Drosophilidae) from Southwest China. Entomotaxonomia 42 (3): 206-226. 2020 [222] doi: 10.11680/entomotax.2020024

CHINA (Yunnan*)

Amiota obtusa Y. L. Wang, H. L. Cao \& H. W. Chen [New Species] IUCN: NE; Evidence: Morphology

WANG Ya-lian, CAO Hui-luo, CHEN Hong-wei, Molecular phylogeny and species delimitation of Amiota alboguttata and Amiota basdeni species groups (Diptera: Drosophilidae) from East Asia. Zoological Journal of the Linnean Society 189 (4): 1370-1397. 2020 [1383] doi: 10.1093/zoolinnean/zlz132

CHINA (Sichuan*)

Amiota planiceps Y. L. Wang, H. L. Cao \& H. W. Chen [New Species] IUCN: NE; Evidence: Morphology

WANG Ya-lian, CAO Hui-luo, CHEN Hong-wei, Molecular phylogeny and species delimitation of Amiota alboguttata and Amiota basdeni species groups (Diptera: Drosophilidae) from East Asia. Zoological Journal of the Linnean Society 189 (4): 1370-1397. 2020 [1384] doi: 10.1093/zoolinnean/zlz132

CHINA (Sichuan*)

Amiota policladia Y. L. Wang \& H. W. Chen 多枝阿果蝇 [New Species] IUCN: NE; Evidence: Morphology; COI

WANG Ya-lian, CHEN Hong-wei, Eleven new species in the genus Amiota (Diptera: Drosophilidae) from Southwest China. Entomotaxonomia 42 (3): 206-226. 2020 [223] doi: 10.11680/entomotax.2020024

CHINA (Sichuan*)

Amiota scrobicula Y. L. Wang, H. L. Cao \& H. W. Chen [New Species] IUCN: NE; Evidence: Morphology

WANG Ya-lian, CAO Hui-luo, CHEN Hong-wei, Molecular phylogeny and species delimitation of Amiota alboguttata and Amiota basdeni species groups (Diptera: Drosophilidae) from East Asia. Zoological Journal of the Linnean Society 189 (4): 1370-1397. 2020 [1385] doi: 10.1093/zoolinnean/zlz132

CHINA (Sichuan*)

Amiota semiannulata Y. L. Wang \& H. W. Chen 断环阿果蝇

[New Species] IUCN: NE; Evidence: Morphology; COI

WANG Ya-lian, CHEN Hong-wei, Eleven new species in the genus Amiota (Diptera: Drosophilidae) from Southwest China. Entomotaxonomia 42 (3): 206-226. 2020 [216] doi: 10.11680/entomotax.2020024

CHINA (Yunnan*)

Amiota tentacula Y. L. Wang, H. L. Cao \& H. W. Chen [New Species] IUCN: NE; Evidence: Morphology

WANG Ya-lian, CAO Hui-luo, CHEN Hong-wei, Molecular phylogeny and species delimitation of Amiota alboguttata and Amiota basdeni species groups (Diptera: Drosophilidae) from East Asia. Zoological Journal of the Linnean Society 189 (4): 1370-1397. 2020 [1386] doi: 10.1093/zoolinnean/zlz132

CHINA (Sichuan*) 
Amiota uncinata Y. L. Wang \& H. W. Chen 钩叶阿果蝇 [New Species] IUCN: NE; Evidence: Morphology; COI

WANG Ya-lian, CHEN Hong-wei, Eleven new species in the genus Amiota (Diptera: Drosophilidae) from Southwest China. Entomotaxonomia 42 (3): 206-226. 2020 [219] doi: 10.11680/entomotax.2020024

CHINA (Yunnan*)

Amiota xinglaii Y. L. Wang, H. L. Cao \& H. W. Chen [New Species] IUCN: NE; Evidence: Morphology

WANG Ya-lian, CAO Hui-luo, CHEN Hong-wei, Molecular phylogeny and species delimitation of Amiota alboguttata and Amiota basdeni species groups (Diptera: Drosophilidae) from East Asia. Zoological Journal of the Linnean Society 189 (4): 1370-1397. 2020 [1387] doi: 10.1093/zoolinnean/zlz132

CHINA (Yunnan*)

Colocasiomyia liae R. J. Jiao, \& J. J. Gao [New Species] IUCN: NE; Evidence: Morphology; COI

JIAO Run-jie, BAI Li-hong, GAO Jian-jun, Descriptions of two new species of the genus Colocasiomyia (Diptera, Drosophilidae) breeding on Rhaphidophora host plants in Yunnan, China. ZooKeys 968: 127-141. 2020 [137] doi: doi: 10.3897/zookeys.968.56677

CHINA (Yunnan*)

Colocasiomyia todai R. J. Jiao, \& J. J. Gao [New Species] IUCN: NE; Evidence: Morphology; COI

JIAO Run-jie, BAI Li-hong, GAO Jian-jun, Descriptions of two new species of the genus Colocasiomyia (Diptera, Drosophilidae) breeding on Rhaphidophora host plants in Yunnan, China. ZooKeys 968: 127-141. 2020 [134] doi: doi: 10.3897/zookeys.968.56677

CHINA (Yunnan*)

Scaptodrosophila abdentata Y. Lin \& H. W. Chen [New Species] IUCN: NE; Evidence: Morphology; COI

LIN Yong, CHEN Hong-wei, The genus Scaptodrosophila Duda (Diptera, Drosophilidae), part III: the riverata species group from China, with morphological and molecular evidence for five new species. ZooKeys 937: 139-162. 2020 [151] doi: 10.3897/zookeys.937.49794

CHINA (Guangdong*, Hainan, Yunnan, Xizang)
Scaptodrosophila platyrhina Y. Lin \& H. W. Chen [New Species] IUCN: NE; Evidence: Morphology; COI

LIN Yong, CHEN Hong-wei, The genus Scaptodrosophila Duda (Diptera, Drosophilidae), part III: the riverata species group from China, with morphological and molecular evidence for five new species. ZooKeys 937: 139-162. 2020 [153] doi: 10.3897/zookeys.937.49794

CHINA (Yunnan*)

Scaptodrosophila serrateifoliacea Y. Lin \& H. W. Chen [New Species] IUCN: NE; Evidence: Morphology; COI

LIN Yong, CHEN Hong-wei, The genus Scaptodrosophila Duda (Diptera, Drosophilidae), part III: the riverata species group from China, with morphological and molecular evidence for five new species. ZooKeys 937: 139-162. 2020 [155] doi: 10.3897/zookeys.937.49794

CHINA (Yunnan*)

Scaptodrosophila sinuata Y. Lin \& H. W. Chen [New Species] IUCN: NE; Evidence: Morphology; COI

LIN Yong, CHEN Hong-wei, The genus Scaptodrosophila Duda (Diptera, Drosophilidae), part III: the riverata species group from China, with morphological and molecular evidence for five new species. ZooKeys 937: 139-162. 2020 [156] doi: 10.3897/zookeys.937.49794

CHINA (Liaoning*)

Stegana (Steganina) alianya Y. Zhang \& H. W. Chen [New Species] IUCN: NE; Evidence: Morphology

WANG Ya-lian, LU Jin-ming, ZHANG Yuan, CHEN Hongwei, Molecular phylogeny, species delimitation and biogeographic history of the Stegana (Steganina) shirozui species group (Diptera: Drosophilidae) from East Asia. Zoological Journal of the Linnean Society 1-19. 2020 [7] doi: $10.1093 /$ zoolinnean/zlaa118

CHINA (Yunnan*)

Stegana (Steganina) diodonta Y. Zhang \& H. W. Chen [New Species] IUCN: NE; Evidence: Morphology

WANG Ya-lian, LU Jin-ming, ZHANG Yuan, CHEN Hongwei, Molecular phylogeny, species delimitation and biogeographic history of the Stegana (Steganina) shirozui species group (Diptera: Drosophilidae) from East Asia. Zoological Journal of the Linnean Society 1-19. 2020 [9] doi: $10.1093 /$ zoolinnean/zlaa118

CHINA (Yunnan*) 
Stegana (Steganina) zebromyia Y. Zhang \& H. W. Chen [New Species] IUCN: NE; Evidence: Morphology

WANG Ya-lian, LU Jin-ming, ZHANG Yuan, CHEN Hong -wei, Molecular phylogeny, species delimitation and biogeographic history of the Stegana (Steganina) shirozui species group (Diptera: Drosophilidae) from East Asia. Zoological Journal of the Linnean Society 1-19. 2020 [11] doi: 10.1093/zoolinnean/zlaa1 18

CHINA (Yunnan*)

Stegana (Steganina) zopheria Y. Zhang \& H. W. Chen [New Species] IUCN: NE; Evidence: Morphology

WANG Ya-lian, LU Jin-ming, ZHANG Yuan, CHEN Hong -wei, Molecular phylogeny, species delimitation and biogeographic history of the Stegana (Steganina) shirozui species group (Diptera: Drosophilidae) from East Asia. Zoological Journal of the Linnean Society 1-19. 2020 [12] doi: 10.1093/zoolinnean/zlaa1 18

CHINA (Yunnan*)

Scaptodrosophila tanyrhina Y. Lin \& H. W. Chen [New Species] IUCN: NE; Evidence: Morphology; COI

LIN Yong, CHEN Hong-wei, The genus Scaptodrosophila Duda (Diptera, Drosophilidae), part III: the riverata species group from China, with morphological and molecular evidence for five new species. ZooKeys 937: 139-162. 2020 [158] doi: 10.3897/zookeys.937.49794

CHINA (Yunnan*)

\section{Empididae 舞虬 科}

Calohilara Yang, Zhang, Yao \& Zhang 弯脉喜舞虻属 [New Genus] IUCN: NE; Evidence: Morphology

DING Shuang-mei, HE Dong-yue, LIN Chen, YANG Ding, Calohilara (Diptera: Empididae), newly recorded from China with description of one new species. Entomotaxonomia 42 (4): 270-274. 2020 [271] doi: 10.11680/entomotax.2020030

MYANMAR (Kambaiti*), CHINA (Xizang)

Calohilara tibetensis S. M. Ding, D. Y. He, C. Lin \& D. Yang 西藏弯脉喜舞虻 [New Species] IUCN: NE; Evidence: Morphology

DING Shuang-mei, HE Dong-yue, LIN Chen, YANG Ding, Calohilara (Diptera: Empididae), newly recorded from China with description of one new species.
Entomotaxonomia 42 (4): 270-274. 2020 [272] doi: 10.11680/entomotax.2020030

CHINA (Xizang*)

Syneches medoganus C. J. Zhao, S. M. Ding, C. Lin \& D. Yang 墨脱柄驼舞虬 [New Species] IUCN: NE; Evidence: Morphology

ZHAO Chen-jing, DING Shuang-mei, LIN Chen, YANG Ding, Two new species of Syneches (Diptera: Empididae) from Tibet, China. Entomotaxonomia 42 (3): 200-205. 2020 [201] doi: 10.11680/entomotax.2020019

CHINA (Xizang*)

Syneches nigritibia C. J. Zhao, S. M. Ding, C. Lin \& D. Yang 黑 胫柄驼舞虻 [New Species] IUCN: NE; Evidence: Morphology

ZHAO Chen-jing, DING Shuang-mei, LIN Chen, YANG Ding, Two new species of Syneches (Diptera: Empididae) from Tibet, China. Entomotaxonomia 42 (3): 200-205. 2020 [203] doi: 10.11680/entomotax.2020019

CHINA (Xizang*)

\section{Lauxaniidae 缟蝇 科}

Cestrotus abdominalis W. L. Li, L. Qi \& D. Yang [New Species] IUCN: NE; Evidence: Morphology

LI Wen-liang, QI Ling, YANG Ding, Four new species of the subfamily Homoneurinae (Diptera, Lauxaniidae) from southwestern China. ZooKeys 953: 119-136. 2020 [122] doi: 10.3897/zookeys.956.53976

CHINA (Yunnan*)

Cestrotus albifacies W. L. Li, L. Qi \& D. Yang [New Species] IUCN: NE; Evidence: Morphology

LI Wen-liang, QI Ling, YANG Ding, Four new species of the subfamily Homoneurinae (Diptera, Lauxaniidae) from southwestern China. ZooKeys 953: 119-136. 2020 [126] doi: 10.3897/zookeys.956.53976

CHINA (Yunnan*)

Minettia (Minettiella) dashahensis W. L. Li, X. L. Chen \& D. Yang [New Species] IUCN: NE; Evidence: Morphology

LI Wen-liang, CHEN Xu-long, YANG Ding, Four new species of the subgenus Minettiella from China (Diptera, Lauxaniidae, Minettia). ZooKeys 932: 93-111. 2020 [97] doi: 
10.3897/zookeys.932.50763

CHINA (Guizhou*)

Minettia (Minettiella) longispina W. L. Li, X. L. Chen \& D. Yang [New Species] IUCN: NE; Evidence: Morphology

LI Wen-liang, CHEN Xu-long, YANG Ding, Four new species of the subgenus Minettiella from China (Diptera, Lauxaniidae, Minettia). ZooKeys 932: 93-111. 2020 [100] doi: $10.3897 /$ zookeys.932.50763

CHINA (Guizhou*)

Minettia (Minettiella) membranacea W. L. Li, X. L. Chen \& D. Yang [New Species] IUCN: NE; Evidence: Morphology

LI Wen-liang, CHEN Xu-long, YANG Ding, Four new species of the subgenus Minettiella from China (Diptera, Lauxaniidae, Minettia). ZooKeys 932: 93-111. 2020 [104] doi: $10.3897 /$ zookeys.932.50763

CHINA (Yunnan*)

Minettia (Minettiella) zhaotongensis W. L. Li, X. L. Chen \& D. Yang [New Species] IUCN: NE; Evidence: Morphology

LI Wen-liang, CHEN Xu-long, YANG Ding, Four new species of the subgenus Minettiella from China (Diptera, Lauxaniidae, Minettia). ZooKeys 932: 93-111. 2020 [107] doi: $10.3897 /$ zookeys.932.50763

CHINA (Yunnan*)

Noeetomima hongshanensis W. L. Li, X. L. Chen \& D. Yang [New Species] IUCN: NE; Evidence: Morphology

LI Wen-liang, CHEN Xu-long, YANG Ding, Five new species of the genus Noeetomima Enderlein (Diptera: Lauxaniidae) from China, with a key to world species.

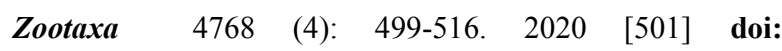
10.11646/zootaxa.4768.4.3

CHINA (Yunnan *, Sichuan)

Noeetomima huzhengkuni L. Shi \& M. Liu [New Species] IUCN: NE; Evidence: Morphology

SHI Li, LIU Miao, HU Zheng-kun, A new species of the genus Noeetomima Enderlein (Diptera, Lauxaniidae) from Guizhou, China with a key to worldwide species. ZooKeys 1000: 107-123. 2020 [113] doi: 10.3897/zookeys.1000.57577

CHINA (Guizhou*)

Noeetomima lijiangensis W. L. Li, X. L. Chen \& D. Yang [New Species] IUCN: NE; Evidence: Morphology
LI Wen-liang, CHEN Xu-long, YANG Ding, Five new species of the genus Noeetomima Enderlein (Diptera: Lauxaniidae) from China, with a key to world species. Zootaxa 4768 (4): 499-516. 2020 [504] doi: 10.11646/zootaxa.4768.4.3

CHINA (Yunnan *, Sichuan)

Noeetomima liui W. L. Li, X. L. Chen \& D. Yang [New Species] IUCN: NE; Evidence: Morphology

LI Wen-liang, CHEN Xu-long, YANG Ding, Five new species of the genus Noeetomima Enderlein (Diptera: Lauxaniidae) from China, with a key to world species. Zootaxa 4768 (4): 499-516. 2020 [507] doi: 10.11646/zootaxa.4768.4.3

CHINA (Yunnan*)

Noeetomima trisurstyla W. L. Li, X. L. Chen \& D. Yang [New Species] IUCN: NE; Evidence: Morphology

LI Wen-liang, CHEN Xu-long, YANG Ding, Five new species of the genus Noeetomima Enderlein (Diptera: Lauxaniidae) from China, with a key to world species. Zootaxa 4768 (4): 499-516. 2020 [509] doi: 10.11646/zootaxa.4768.4.3

CHINA (Guangxi*)

Noeetomima zhangae W. L. Li, X. L. Chen \& D. Yang [New Species] IUCN: NE; Evidence: Morphology

LI Wen-liang, CHEN Xu-long, YANG Ding, Five new species of the genus Noeetomima Enderlein (Diptera: Lauxaniidae) from China, with a key to world species. Zootaxa 4768 (4): 499-516. 2020 [512] doi: 10.11646/zootaxa.4768.4.3

CHINA (Yunnan*)

Noonamyia bicolour W. L. Li, L. Qi \& D. Yang [New Species] IUCN: NE; Evidence: Morphology

LI Wen-liang, QI Ling, YANG Ding, Three new species of the genus Noonamyia Stuckenberg 1971 (Diptera: lauxaniidae) from China. Oriental Insects 1-14. 2020 [3] doi: 10.1080/00305316.2020.1754955

CHINA (Xizang*)

Noonamyia brevisurstyla W. L. Li, L. Qi \& D. Yang [New Species] IUCN: NE; Evidence: Morphology

LI Wen-liang, QI Ling, YANG Ding, Three new species of the genus Noonamyia Stuckenberg 1971 (Diptera: lauxaniidae) from China. Oriental Insects 1-14. 2020 [6] doi: 10.1080/00305316.2020.1754955

CHINA (Yunnan*) 


\section{IUCN: NE; Evidence: Morphology}

LI Wen-liang, QI Ling, YANG Ding, Three new species of the genus Noonamyia Stuckenberg 1971 (Diptera: lauxaniidae) from China. Oriental Insects 1-14. 2020 [10] doi: $10.1080 / 00305316.2020 .1754955$

CHINA (Yunnan*)

Phobeticomyia motuoensis W. L. Li, L. Qi \& D. Yang [New Species] IUCN: NE; Evidence: Morphology

LI Wen-liang, QI Ling, YANG Ding, Four new species of the subfamily Homoneurinae (Diptera, Lauxaniidae) from southwestern China. ZooKeys 953: 119-136. 2020 [129] doi: 10.3897/zookeys.956.53976

\section{CHINA (Xizang*)}

Prosopophorella longa W. L. Li, L. Qi \& D. Yang [New Species] IUCN: NE; Evidence: Morphology

LI Wen-liang, QI Ling, YANG Ding, Four new species of the subfamily Homoneurinae (Diptera, Lauxaniidae) from southwestern China. ZooKeys 953: 119-136. 2020 [132] doi: 10.3897/zookeys.956.53976

CHINA (Xizang*)

\section{Limoniidae 沼大蚊 科}

Geranomyia jiulongensis X. Y. Qian \& X. Zhang 九龙长唇大蚊

[New Species] IUCN: NE; Evidence: Morphology

QIAN Xing-yang, ZHANG Xiao, Two new Geranomyia Haliday (Diptera, Limoniidae) crane flies from Mount Jiulong in China, with an updated key to Chinese species. ZooKeys 953: 105-118. 2020 [110] doi: 10.3897/zookeys.953.49557

CHINA (Zhejiang*)

Geranomyia subablusa X. Y. Qian \& X. Zhang [New Species] IUCN: NE; Evidence: Morphology

QIAN Xing-yang, ZANG Xiao, Two new Geranomyia Haliday (Diptera, Limoniidae) crane flies from Mount Jiulong in China, with an updated key to Chinese species. ZooKeys 953: 105-118. 2020 [112] doi: 10.3897/zookeys.953.49557

CHINA (Zhejiang*)

Limonia medexocha J. L. Ren \& D. Yang 中突沼大蚊 [New Species] IUCN: NE; Evidence: Morphology
REN Jin-long, YANG Ding, Two new species of Limonia Meigen, 1803 from Northwest China (Diptera, Limoniidae). ZooKeys 971: 31-58. $2020 \quad$ [37] doi: 10.3897/zookeys.971.35875

CHINA (Ningxia*, Sichuan)

Limonia subcosta J. L. Ren \& D. Yang 缘脉沼大蚊 [New Species] IUCN: NE; Evidence: Morphology

REN Jin-long, YANG Ding, Two new species of Limonia Meigen, 1803 from Northwest China (Diptera, Limoniidae). ZooKeys 971: 31-58. 2020 [46] doi: 10.3897/zookeys.971.35875

CHINA (Shaanxi*)

Metalimnobia (Metalimnobia) bicolor Y. Jiang \& X. Zhang [New Species] IUCN: NE; Evidence: Morphology

JIANG Ye, ZHANG Xiao, New Metalimnobia crane flies (Diptera, Limoniidae) from China with an update of species distributions. ZooKeys 1008: 93-105. 2020 [95] doi: 10.3897/zookeys.1008.60704

CHINA (Sichuan*)

Metalimnobia (Metalimnobia) caudifusca Y. Jiang \& X. Zhang [New Species] IUCN: NE; Evidence: Morphology

JIANG Ye, ZHANG Xiao, New Metalimnobia crane flies (Diptera, Limoniidae) from China with an update of species distributions. ZooKeys 1008: 93-105. 2020 [100] doi: 10.3897/zookeys. 1008.60704

CHINA (Xizang*)

\section{Lonchaeidae 尖尾蝇科}

Silba erecta I. MacGowan \& K. Arimoto [New Species] IUCN: NE; Evidence: Morphology

ARIMOTO Kôichi, MACGOWAN Iain, SU Zhi-hui, New data on lance flies (Diptera, Lonchaeidae) associated with figs (Moraceae, Ficus spp.) in Japan and Taiwan, with descriptions of two new species of the genus Silba Macquart. Journal of Asia-Pacific Entomology 23 (2): 364-370. 2020 [365] doi: 10.1016/j.aspen.2019.11.007

CHINA (Taiwan*) 
Phaonia liujiayni W. Q. Xue \& J. Du [New Species] IUCN: NE; Evidence: Morphology

XUE Wan-qi, DU Jing, Five new species of Phaonia Robineau-Desvoidy (Diptera: Muscidae) from China.

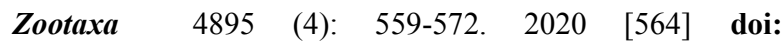
10.11646/zootaxa.4895.4.6

CHINA (Ningxia*)

Phaonia sunqiia W. Q. Xue \& J. Du [New Species] IUCN: NE; Evidence: Morphology

XUE Wan-qi, DU Jing, Five new species of Phaonia Robineau-Desvoidy (Diptera: Muscidae) from China. $\begin{array}{llllll}\text { Zootaxa } & 4895 & \text { (4): } & 559-572 . & 2020 & \text { [560] doi: }\end{array}$ 10.11646/zootaxa.4895.4.6

CHINA (Hebei*)

Phaonia tianmushanensis W. Q. Xue \& J. Du [New Species] IUCN: NE; Evidence: Morphology

XUE Wan-qi, DU Jing, Five new species of Phaonia Robineau-Desvoidy (Diptera: Muscidae) from China. $\begin{array}{llllll}\text { Zootaxa } & 4895 & \text { (4): } & 559-572 . & 2020 & \text { [568] doi: }\end{array}$ 10.11646/zootaxa.4895.4.6

CHINA (Zhejiang*)

Phaonia wangpingga W. Q. Xue \& J. Du [New Species] IUCN: NE; Evidence: Morphology

XUE Wan-qi, DU Jing, Five new species of Phaonia Robineau-Desvoidy (Diptera: Muscidae) from China. $\begin{array}{llllll}\text { Zootaxa } & 4895 & \text { (4): } & 559-572 . & 2020 & \text { [566] doi: }\end{array}$ 10.11646/zootaxa.4895.4.6

CHINA (Zhejiang*)

Phaonia zhangjunyui W. Q. Xue \& J. Du [New Species] IUCN: NE; Evidence: Morphology

XUE Wan-qi, DU Jing, Five new species of Phaonia Robineau-Desvoidy (Diptera: Muscidae) from China $\begin{array}{llllll}\text { Zootaxa } & 4895 & \text { (4): } & 559-572 . & 2020 & \text { [562] doi: }\end{array}$ 10.11646/zootaxa.4895.4.6

CHINA (Sichuan*)

\section{Oestridae 狂蝇 科}

Portschinskia burmensis X. Y. Li, T. Pape \& D. Zhang [New Species] IUCN: NE; Evidence: Morphology

LI Xin-yu, PAPE Thomas, ZHANG Dong, Taxonomy, phylogeny and evolution of the bumblebee bot flies
(Oestridae: Hypodermatinae: Portschinskia). Zoological Journal of the Linnean Society 1-32. 2020 [8] doi: 10.1093/zoolinnean/zlz176/5854325

MYANMAR (Adung Valley*), CHINA (Yunnan)

Portschinskia sichuanensis X. Y. Li, T. Pape \& D. Zhang [New Species] IUCN: NE; Evidence: Morphology

LI Xin-yu, PAPE Thomas, ZHANG Dong, Taxonomy, phylogeny and evolution of the bumblebee bot flies (Oestridae: Hypodermatinae: Portschinskia). Zoological Journal of the Linnean Society 1-32. 2020 [22] doi: 10.1093/zoolinnean/zlz176/5854325

CHINA (Sichuan*)

Portschinskia xizangensis X. Y. Li, T. Pape \& D. Zhang [New Species] IUCN: NE; Evidence: Morphology

LI Xin-yu, PAPE Thomas, ZHANG Dong, Taxonomy, phylogeny and evolution of the bumblebee bot flies (Oestridae: Hypodermatinae: Portschinskia). Zoological Journal of the Linnean Society 1-32. 2020 [24] doi: 10.1093/zoolinnean/zlz176/5854325

CHINA (Xizang*)

Portschinskia yunnanensis X. Y. Li, T. Pape \& D. Zhang [New Species] IUCN: NE; Evidence: Morphology

LI Xin-yu, PAPE Thomas, ZHANG Dong, Taxonomy, phylogeny and evolution of the bumblebee bot flies (Oestridae: Hypodermatinae: Portschinskia). Zoological Journal of the Linnean Society 1-32. 2020 [26] doi: 10.1093/zoolinnean/zlz176/5854325

CHINA (Yunnan*)

\section{Phoridae 蚤蝇科}

Epicnemis linguiformis M. Y. Chu \& G. C. Liu [New Species] IUCN: NE; Evidence: Morphology

CHU Meng-ying, LIU Guang-chun, First record of genus Epicnemis Borgmeier, 1962 (Aenigmatiinae: Phoridae: Diptera) from China, with description of a new species. $\begin{array}{llllll}\text { Zootaxa } & 4772 & \text { (3): } & 593-596 . & 2020 & \text { [593] doi: }\end{array}$ 10.11646/zootaxa.4772.3.9

CHINA (Jiangxi*)

Sinogodavaria G. C. Liu [New Genus] IUCN: NE; Evidence: Morphology

LIU Guang-chun, A new genus of the Hypocera group (Diptera, 
Phoridae), with descriptions of two new species from China. ZooKeys 932: 113-127. 2020 [114] doi: 10.3897/zookeys.932.38970

CHINA (Liaoning*, Hebei, Shaanxi, Sichuan)

Sinogodavaria multiformis G. C. Liu [New Species] IUCN: NE; Evidence: Morphology

LIU Guang-chun, A new genus of the Hypocera group (Diptera, Phoridae), with descriptions of two new species from China. ZooKeys 932: 113-127. 2020 [116] doi: 10.3897/zookeys.932.38970

CHINA (Liaoning*, Hebei, Shaanxi)

Sinogodavaria tenebrosa G. C. Liu [New Species] IUCN: NE; Evidence: Morphology

LIU Guang-chun, A new genus of the Hypocera group (Diptera, Phoridae), with descriptions of two new species from China. ZooKeys 932: 113-127. 2020 [120] doi: 10.3897/zookeys.932.38970

CHINA (Shaanxi*)

\section{Sarcophagidae 麻蝇 科}

Sarcophaga (Hoa) membranijuxta C. Wang, W. Q. Xue, D. Zhang \& T. Pape [New Species] IUCN: NE; Evidence: Morphology

WANG Chao, XUE Wan-qi, ZHANG Dong, PAPE Thomas, A new species of Sarcophaga Meigen subgenus Hoa Rohdendorf (Diptera: Sarcophagidae). Zootaxa 4821 (3): 585-593. 2020 [589] doi: 10.11646/zootaxa.4821.3.9

CHINA (Hainan*, Guangxi)

\section{Syrphidae 蚜蝇科}

Criorhina rostrata H. Li, K. K. Huo \& B. G. Li [New Species] IUCN: NE; Evidence: Morphology

LI Hu, HUO Ke-ke, LI Bao-Guo, A new species of the flower fly genus Criorhina Meigen (Diptera: Syrphidae) from mainland China. Zootaxa 4803 (1): 169-176. 2020 [170] doi: 10.11646/zootaxa.4803.1.9

CHINA (Henan*)

Eumerus alxaensis K. K. Huo \& A. P. Liu 阿拉善平颜蚜蝇 [New Species] IUCN: NE; Evidence: Morphology
SONG Mi-xia, LIU Ai-ping, HUO Ke-ke, SHI Li, GAN Lin, Two new species in the genus Eumerus Meigen (Diptera: Syrphidae) from Inner Mongolia, China. Entomotaxonomia 42 (4): 275-281. 2020 [276] doi:

10.11680/entomotax.2020032

CHINA (Inner Mongolia*)

Eumerus seximaculatum K. K. Huo \& A. P. Liu 六斑平颜蚜蝇

[New Species] IUCN: NE; Evidence: Morphology

SONG Mi-xia, LIU Ai-ping, HUO Ke-ke, SHI Li, GAN Lin, Two new species in the genus Eumerus Meigen (Diptera: Syrphidae) from Inner Mongolia, China. Entomotaxonomia 42 (4): 275-281. 2020 [278] doi:

10.11680/entomotax.2020032

CHINA (Inner Mongolia*)

\section{Tachinidae 寄蝇科}

Admontia quwi Y. Z. Huang \& T. Tachi [New Species] IUCN: NE; Evidence: Morphology

HUANG Yu-zen, TACHI Takuji, A new species of Admontia Brauer \& Bergenstamm from Taiwan (Diptera: Tachinidae: Exoristinae: Blondeliini). Journal of Asia-Pacific Entomology 24: 151-157. 2020 [155] doi: 10.1016/j.aspen.2020.11.011

CHINA (Taiwan*)

Linnaemya (s.str.) flavifemur Y. Z. Zhang 黄股短须寄蝇 [New Species] IUCN: NE; Evidence: Morphology

ZHANG Yi-zhuo, HAO Bo, ZHANG Chun-tian, One new species, two newly recorded genera and four newly recorded species of Tachinidae (Diptera) from Liaoning, China. Entomotaxonomia 42 (2): 122-129. 2020 [123] doi: 10.11680/entomotax.2020012

CHINA (Liaoning*)

\section{Tipulidae 大 蚊 科}

Nephrotoma beibengensis Q. C. Yang, Q. F. Liu, X. Y. Liu \& D. Yang 背崩短柄大蚊 [New Species] IUCN: NE; Evidence: Morphology

YANG Qi-cheng, LIU Qi-fei, PAN Zhao-hui, LIU Xiao-yan, YANG Ding, Nephrotoma Meigen (Diptera, Tipulidae) from Xizang Autonomous Region, China. ZooKeys 973: 123-151. 2020 [125] doi: $10.3897 /$ zookeys.973.46384 


\section{CHINA (Xizang*)}

Nephrotoma hanae Q. C. Yang, Q. F. Liu, X. Y. Liu \& D Yang 韩氏短柄大蚊 [New Species] IUCN: NE; Evidence: Morphology

YANG Qi-cheng, LIU Qi-fei, PAN Zhao-hui, LIU Xiao-yan, YANG Ding, Nephrotoma Meigen (Diptera, Tipulidae) from Xizang Autonomous Region, China. ZooKeys 973: 123-151. 2020 [138] doi: 10.3897/zookeys.973.46384

CHINA (Xizang*)

Tipula (Formotipula) gongshanensis Q. L. Men 贡山丽大蚊 [New Species] IUCN: NE; Evidence: Morphology

MEN Qiu-lei, Two new species in the subgenus Formotipula Matsumura (Diptera: Tipulidae: Tipula) from China. Entomotaxonomia 42 (4): 282-297. 2020 [283] doi: 10.11680/entomotax.2020034

CHINA (Yunnan*)

Tipula (Formotipula) medogensis Q. L. Men 墨脱丽大蚊 [New Species] IUCN: NE; Evidence: Morphology

MEN Qiu-lei, Two new species in the subgenus Formotipula Matsumura (Diptera: Tipulidae: Tipula) from China Entomotaxonomia 42 (4): 282-297. 2020 [286] doi: 10.11680/entomotax.2020034

CHINA (Xizang*)

Tipula (Sivatipula) pianmaensis Q. L. Men 片马长角大蚊 [New Species] IUCN: NE; Evidence: Morphology

MEN Qiu-lei, Three new species of the subgenus Tipula (Sivatipula) from Yunnan and Tibet, China (Diptera: Tipulidae), with an updated key for Chinese species. Zoological Systematics 45 (1): 59-68. 2020 [60] doi: 10.11865/zs.202006

CHINA (Yunnan*)

Tipula (Sivatipula) podenasi Q. L. Men [New Species] IUCN: NE; Evidence: Morphology

MEN Qiu-lei, Three new species of the subgenus Tipula (Sivatipula) from Yunnan and Tibet, China (Diptera: Tipulidae), with an updated key for Chinese species. Zoological Systematics 45 (1): 59-68. 2020 [65] doi: 10.11865/zs.202006

CHINA (Yunnan*)
Tipula (Sivatipula) pseudofilicornis Q. L. Men [New Species] IUCN: NE; Evidence: Morphology

MEN Qiu-lei, Three new species of the subgenus Tipula (Sivatipula) from Yunnan and Tibet, China (Diptera: Tipulidae), with an updated key for Chinese species. Zoological Systematics 45 (1): 59-68. 2020 [63] doi: 10.11865/zs.202006

CHINA (Xizang*)

\section{List of Journals 杂志参考}

Annales Zoologici Fennici. ISSN: 0003-455X. Finland: 1964-.

Chinese Journal of Frontier Health and Quarantine, 中国国 境卫生检疫杂志. ISSN: 1004-9770. China: Beijing 1978-.

Chinese Journal of Vector Biology and Control, 中国媒介生 物学及控制杂志. ISSN: 1003-4692. China: Beijing 1985-.

Diversity. ISSN: 1424-2818. Switzerland: 2009-.

Entomological Science. ISSN: 1343-8786. Japan: 1998-

Entomotaxonomia, 昆虫分类学报. ISSN: 2095-8609. China: Yangling 1979-.

Journal of Asia-Pacific Entomology. ISSN: 1226-8615. South Korea: 1998-.

Oriental Insects. ISSN: 0030-5316. India: 1967-.

The Pan-Pacific Entomologist. ISSN: 0031-0603. United States: 1924-.

Transactions of the American Entomological Society. ISSN: 0002-8320. United States: 2006-.

ZooKeys. ISSN: 1313-2989. Bulgaria: 2008-.

Zoological Journal of the Linnean Society. ISSN: 0024-4082. England: 1969-.

Zoological Systematics, 动物分类学报. ISSN: 2095-6827. China: Beijing 1964-.

Zootaxa. ISSN: 1175-5326. New Zealand: 2001- 